\title{
High-Order Harmonic Generation from Ions in a Capillary Discharge
}

\author{
David M. Gaudiosi, ${ }^{1}$ Brendan Reagan, ${ }^{2}$ Tenio Popmintchev, ${ }^{1}$ Michael Grisham, ${ }^{2}$ Mark Berrill, ${ }^{2}$ Oren Cohen, ${ }^{1}$ \\ Barry C. Walker, ${ }^{3}$ Margaret M. Murnane, ${ }^{1}$ Henry C. Kapteyn, ${ }^{1}$ and Jorge J. Rocca ${ }^{2}$ \\ NSF ERC for Extreme Ultraviolet Science and Technology \\ ${ }^{1}$ JILA, University of Colorado and National Institute of Standards and Technology, 440 UCB, Boulder, Colorado, 80309-0440, USA \\ ${ }^{2}$ Department of Electrical and Computer Engineering, Colorado State University, Fort Collins, Colorado 80523, USA \\ ${ }^{3}$ Department of Physics and Astronomy, University of Delaware, 223 Sharp Laboratory, Newark, Delaware 19716, USA
}

(Received 19 January 2006; published 25 May 2006)

\begin{abstract}
We demonstrate a significant extension of the high-order harmonic cutoff by using a fully-ionized capillary discharge plasma as the generation medium. The preionized plasma dramatically reduces ionization-induced defocusing and energy loss of the driving laser due to ionization. This allows for significantly higher photon energies, up to $150 \mathrm{eV}$, to be generated from xenon ions, compared with the $70 \mathrm{eV}$ observed previously. We also demonstrate enhancement of the harmonic flux of nearly 2 orders of magnitude at photon energies around $90 \mathrm{eV}$ when the capillary discharge is used to ionize xenon, compared with harmonic generation in a hollow waveguide. The use of a plasma as a medium for highorder harmonic generation shows great promise for extending efficient harmonic generation to much shorter wavelengths using ions.
\end{abstract}

DOI: 10.1103/PhysRevLett.96.203001

PACS numbers: $42.65 . \mathrm{Ky}, 32.80 . \mathrm{Wr}, 42.65 . \mathrm{Re}, 52.35 . \mathrm{Mw}$

High-order harmonic generation (HHG) coherently upconverts laser light from the visible and infrared into the extreme-ultraviolet region of the spectrum. Over the past decade, HHG has been demonstrated as a useful light source for a wide range of applications, such as investigating surface dynamics [1], holographic imaging [2], and more recently for probing static molecular structure $[3,4]$, or internal molecular dynamics. In HHG, the nonlinear interaction between a material, typically a gas [5,6], and an intense laser field produces high-order harmonics of the fundamental laser. The laser field first ionizes the atom or molecule, then accelerates the liberated electron away from the ion, finally generating high-order harmonic photons when the laser field reverses and the oscillating electron recollides with its parent ion. The highest photon energy that can be produced via this interaction is predicted [7,8] by the cutoff rule to be $h \nu_{\max }=I_{\mathrm{p}}+3.17 U_{\mathrm{p}}$, where $I_{\mathrm{p}}$ is the ionization potential of the atom and $U_{\mathrm{p}} \propto I_{\mathrm{L}} \lambda^{2}$ is the ponderomotive energy of the electron in the laser field. Here $I_{\mathrm{L}}$ is the peak laser intensity and $\lambda$ is the wavelength of the driving laser field.

From the cutoff rule, the range of photon energies that are generated in HHG is determined by the laser intensity. However, in most experiments to date, the maximum observed HHG photon energy has been limited not by the available laser intensity, but by the intensity at which the target atoms are nearly completely $(\sim 98 \%)$ ionized-or the "saturation intensity" $I_{\mathrm{S}}\left(<I_{\mathrm{L}}\right)$ of the medium. This is because at near full ionization in a medium, ionizationinduced refraction $[9,10]$ of the laser beam reduces the effective laser intensity compared with what could be obtained in a vacuum. Moreover, reduced coherence lengths also limit the number of atoms contributing to the harmonic emission. To obtain the highest possible photon energy, $I_{\mathrm{s}}$ can be increased either by using a shorter duration laser pulse, or by using atoms with a higherionization potential, both of which allow neutral atoms to survive to a higher laser intensity. Partial phase matching of the harmonic emission in a plasma can also be implemented to increase the harmonic output. Recently, Gibson et al. demonstrated that, by generating harmonics in a gasfilled modulated waveguide, the effects of ionizationinduced refraction and low coherence lengths could be ameliorated somewhat. As a result, substantially higher photon energies were generated from argon ions, at laser intensities well above $I_{\mathrm{s}}$ [11]. However, the effects of ionization-induced refraction of the laser beam are still significant, and lead to significant losses in the waveguide, ultimately limiting the laser intensity and cutoff photon energy.

Thus, no fundamental limits have yet been reached in the highest photon energies obtainable from HHG. To date, harmonics up to $\approx 1 \mathrm{keV}$ have been observed in conventional HHG using 5 fs pulses focused into neutral helium [12], which has an ionization potential of $24.6 \mathrm{eV}$. In principle, HHG from helium ions could extend this cutoff to a photon energy of several keV. However, emission of high-energy photons through ionization of helium results in very low flux because the electron recollision cross section for helium, and thus the effective nonlinear susceptibility of the HHG process, is extremely small. By using larger target ions, it might be possible to obtain much higher harmonic flux at a given photon energy. Xenon has a very large effective susceptibility; to date the highest photon energy generated from xenon with an $800 \mathrm{~nm}$ driving laser was $\sim 70 \mathrm{eV} \mathrm{[13]} \mathrm{and} \mathrm{was} \mathrm{attributed} \mathrm{to} \mathrm{xenon}$ ions. Emission from xenon ions could in principle extend to substantially higher photon energies. However, because 
of the low ionization potential of $\mathrm{Xe}(12.13 \mathrm{eV})$, the large amount of ionization that accompanies HHG leads to significant loss of laser energy, due to ionization loss and ionization-induced defocusing, as well as poor phasematching conditions. Preformed plasma waveguides have been suggested as an attractive medium for high-order harmonic generation that could overcome two of these limitations $[14,15]$.

In this Letter, we address one of the main limitations to generating very high-order harmonics from ions by demonstrating high-order harmonic generation in a capillary discharge plasma. This significantly suppresses energy loss due to ionization-induced defocusing of the laser beam and maintains a high laser intensity in the interaction region. In our work, the observed HHG cutoff in xenon ions was first extended from the previously observed 70 to $90 \mathrm{eV}$ using high-order harmonic generation in a gas-filled waveguide. The observed maximum photon energy was then extended further, to $150 \mathrm{eV}$, by generating the harmonics in a completely ionized xenon plasma column created by a capillary discharge. The observed photon energies of $150 \mathrm{eV}$ from xenon ions far exceeds the accessible photon energies that can be generated either from neutral krypton or xenon atoms. Higher-ionization potential gases such as $\mathrm{Ne}$ and $\mathrm{He}$ can produce harmonic emission at shorter wavelengths However, emission from neutral Ne extends only to $\approx 300 \mathrm{eV}$, and eventually even helium becomes fully ionized at photon energies $\approx 1 \mathrm{keV}$. Therefore, to generate bright, multi-keV photons using high-order harmonic generation, it will be necessary to generate harmonics from ions in a fully or multiply ionized plasma, as we demonstrate here. In addition to the extension of the cutoff photon energy, preionizing with a capillary discharge increases the HHG flux in xenon at $90 \mathrm{eV}$ by nearly 2 orders of magnitude. As a result, use of a capillary discharge plasma as a medium for high-order harmonic generation shows great promise for extending efficient harmonic generation to much shorter wavelengths.

Capillary discharges can generate elongated plasma columns with a concave electron density profile capable of efficiently guiding high intensity laser pulses [15-17] and reducing the effects of plasma defocusing. Moreover, the degree of ionization can be tuned by changing the discharge current. This is important for HHG since the driving laser can then interact with a tailored, preformed, completely ionized plasma, minimizing the plasma defocusing and energy loss due to ionization. Previous work has studied high-order harmonic generation using preformed plasmas created through ablation of a solid [18-20]. However, these experiments do not have a well-characterized target species and thus emission from the neutrals and ions cannot be distinguished. Moreover, these experiments do not benefit from any waveguide effect and as a result, no dramatic extension of cutoff was observed.

The experiment, illustrated schematically in Fig. 1, was conducted by focusing pulses from a $10 \mathrm{~Hz}$ chirped-pulse amplification Ti:sapphire laser system [21] into a plasma

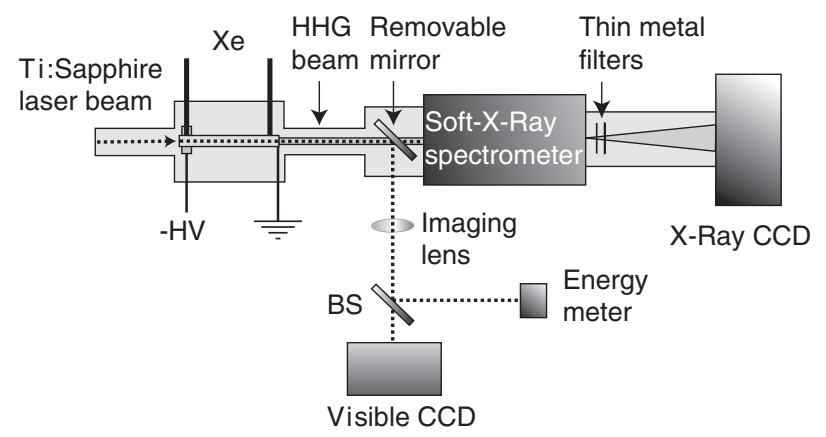

FIG. 1. Schematic of the experimental setup.

column generated by an electrical discharge in a $175 \mu \mathrm{m}$ diameter bore, fused silica capillary. Laser pulses of $28 \mathrm{fs}$ in duration with an energy of $5 \mathrm{~mJ}$ were focused in vacuum to a diameter of $\sim 100 \mu \mathrm{m}$ at the entrance of the capillary. Two holes drilled $3 \mathrm{~mm}$ from the ends of the capillary serve as gas inlets and help maintain a uniform gas pressure in the $4.5 \mathrm{~cm}$ long central region. The front gas inlet was surrounded by a hollow cathode, while the anode consisted of a metal plate at the end of the capillary with a through hole to allow the harmonics to exit. The compact discharge was mounted on an $X-Y$ stage supported by a 2-axis tilt platform to facilitate alignment with the laser pulse. The HHG spectrum was measured using a flat field euv spectrometer and an X-ray CCD camera. Thin metal filters were placed between the spectrometer and the CCD camera to block the fundamental laser light, allow different energy regions of the euv spectrum to be observed, and to calibrate the euv spectrum. By inserting a removable mirror after the capillary, the exiting laser light was directed into an imaging system that measured the spatial mode at the exit of the capillary as well as the pulse energy.

The capillary was filled with 2-4 Torr of xenon gas, and a continuous de simmer current of $\sim 10 \mathrm{~mA}$ was used to maintain a low degree of ionization to predictably initiate the pulsed discharge. By applying a current pulse of varying amplitude with rise time $\sim 350 \mathrm{~ns}$ and a decay constant of $\sim 2 \mu \mathrm{s}$, a preformed plasma consisting of predominantly Xe II or Xe III can be created. The combination of Ohmic heating and heat conduction to the capillary walls creates a plasma column with maximum temperature on axis. Pressure balance gives rise to a concave electron density profile with a minimum on axis constituting an index waveguide [15]. The results reported here were obtained using a 5 A peak current, as measured by a Rogowski coil. The discharge was operated at $10 \mathrm{~Hz}$ and was synchronized to the laser. Time resolved visible spectroscopy on the discharge was performed by replacing the euv spectrometer with a $0.6 \mathrm{~m}$ visible spectrometer and a gated, intensified, diode array.

To maximize the harmonic signal, we optimized the characteristics of the plasma column for optimal HHG cutoff, by adjusting the discharge current, the xenon gas pressure, and the delay of the laser pulse with respect to the rising edge of the discharge current. The HHG emission 
was then monitored, both with and without the discharge. Under optimal conditions, the cutoff energy can be dramatically extended, as shown in Fig. 2. These spectra were obtained at a xenon pressure of 2.9 Torr with the laser being injected $2 \mu \mathrm{s}$ after the onset of current pulse. Without the discharge running, the signal drops by 5 orders of magnitude between 75 and $90 \mathrm{eV}$, where it drops into the noise of our measurement. This large drop in signal over a relatively small energy range is the signature of the cutoff region in HHG. Therefore, without the discharge running, the highest observable harmonic extends to $90 \mathrm{eV}$ in the hollow waveguide. This initial extension of the cutoff compared with past work is due to a partial reduction of ionization-induced defocusing in the guided geometry. The short defocusing length in a gas jet, given by $L_{\mathrm{D}}=$ $\lambda n_{\mathrm{c}} / 2 n_{\mathrm{e}}$, where $n_{\mathrm{e}}$ is the electron density and $n_{\mathrm{c}}$ is the critical density, would otherwise defocus the laser beam and lead to a reduction in intensity in a distance of under $7 \mathrm{~mm}[10,11]$. When the discharge is initiated to create the optimal ion population and profile in the waveguide prior to the arrival of the laser, the observed cutoff extends still further - to $150 \mathrm{eV}$. This represents greater than a factor of 2 extension in the observed cutoff compared with previous work. The presence of the discharge also enhances the HHG emission by nearly 2 orders of magnitude for photon energies around the cutoff without the discharge $(\sim 90 \mathrm{eV})$.

The extension of the cutoff and the enhancement of the harmonic flux in the presence of the discharge plasma are due to a concave plasma density profile that is completely ionized on axis prior to the arrival of the laser pulse. This density profile aids in guiding the driving laser pulse and

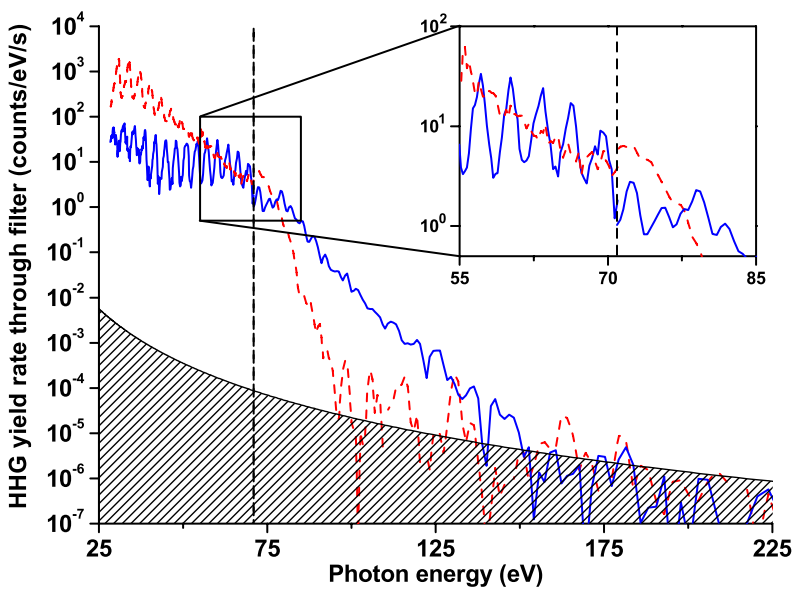

FIG. 2 (color online). High-order harmonic spectra on a log scale with (solid blue) and without (dashed red) the preionizing discharge. The vertical dotted line shows the split between data taken through thin aluminum filters (low energy) and thin aluminum-coated zirconium filters (high energy). The hatched area represents our noise floor. Inset: harmonic generation in the 55-85 eV spectral region, showing that individual harmonic peaks are much better resolved when the discharge is used to preionize the xenon, thereby reducing ionization-induced modulation of the laser. mitigates ionization-induced refraction, while the completely ionized media will reduce the laser energy lost to ionization. Our measurements show that the transmission efficiency of the fundamental light through the waveguide increases from $31 \%$ without the discharge to $62 \%$ with the discharge on. These effects result in a higher laser intensity at the exit of the capillary and therefore, from the cutoff rule, lead to higher harmonic photon energies.

Another prominent feature in the data of Fig. 2 (and magnified in the inset) is the fact that, in the $55-85 \mathrm{eV}$ spectral region, the individual harmonic peaks are much better resolved when the discharge is used to preionize the xenon. Without the discharge, the lack of resolved harmonic peaks results from self-phase modulation of the fundamental laser pulse that would be expected to accompany the rapid ionization of the neutral xenon by the laser. When the discharge preionizes the xenon, phase modulation of the laser is dramatically reduced, and occurs only through ionization of the ions. This reduced phase modulation leads to better resolved harmonic peaks. Spectral measurements of the laser after the capillary show significant spectral broadening and blueshifting without the discharge, as shown in Fig. 3. In contrast, when the discharge is running, the fundamental laser spectrum shows a very small change in spectrum from that observed from an evacuated capillary.

Yet another feature of the data of Fig. 2 that is consistent with our understanding is that, at the lowest photon energies $(<50 \mathrm{eV})$, the harmonic signal decreases substantially with the discharge. In this spectral region, harmonic emission without the discharge is primarily from ionization of neutrals. However, when the discharge is initiated under our conditions, the neutrals are depleted. Although HHG from ions will also generate photons in this energy range, the emission occurs in a plasma with significantly shorter coherence length $(\sim 1 \mathrm{~mm}$ with the discharge on as op-

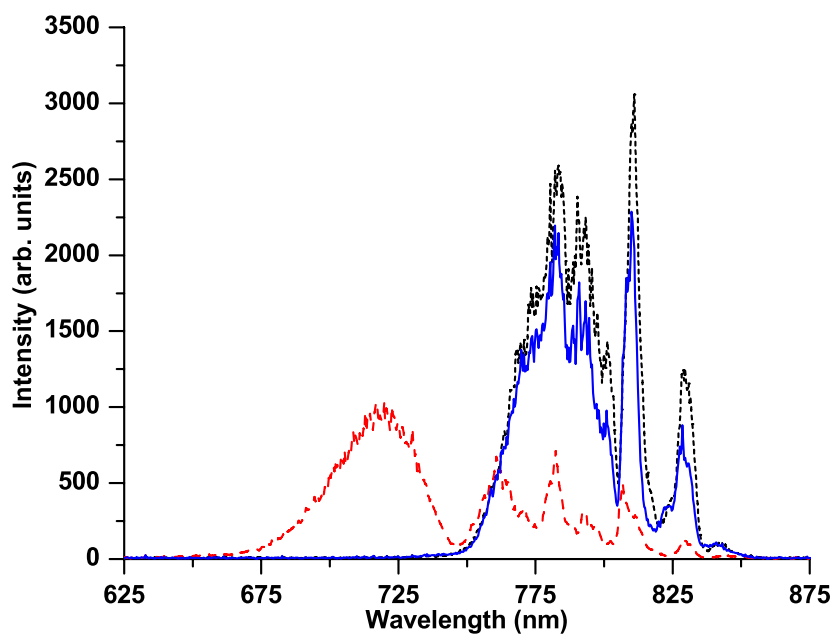

FIG. 3 (color online). Spectral measurements of the exiting fundamental light for an evacuated capillary (dotted black), gasfilled capillary with no discharge (dashed red), and gas-filled capillary with discharge (solid blue). 


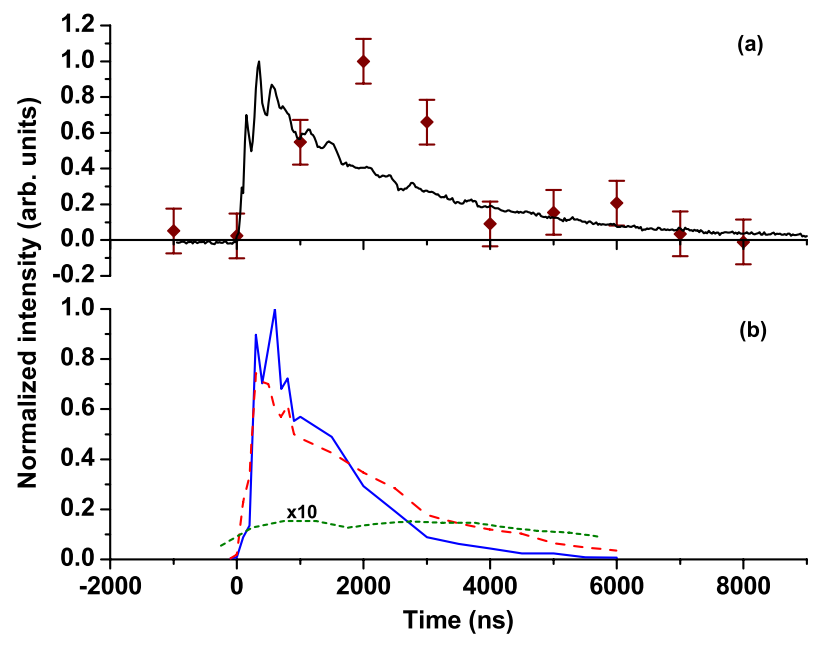

FIG. 4 (color online). (a) Time evolution of the harmonic signal at $120 \mathrm{eV}$ (red diamonds). The current pulse is shown for reference (black). (b) Time evolution of the visible emission lines from Xe I $(823.16 \mathrm{~nm})$ (dotted green), Xe II (418.01 nm) (dashed red), and Xe III (410.91 nm) (solid blue).

posed to $\sim 5 \mathrm{~mm}$ without the discharge for the 19th harmonic) than in a neutral gas, thereby reducing the efficiency.

Figure 4 further illustrates the optimum plasma conditions for harmonic generation. Figure 4(a) shows the harmonic signal at $120 \mathrm{eV}$, together with the current pulse temporal profile. Figure 4(b) shows selected visible emission lines from Xe I $(823.16 \mathrm{~nm})$, Xe II $(418.01 \mathrm{~nm})$, and Xe III $(410.91 \mathrm{~nm})$ on the same time scale. The intensity of this harmonic is zero before the onset of the current pulse, increases after this onset, reaches a peak at a delay of 2 us, and then quickly decays back to zero. This clearly shows the advantages of generating harmonics in a capillary discharge. It is possible that the delay of the maximum HHG signal is due to the presence of plasma nonuniformities caused by the fast rise time of the current pulse. Furthermore, early in the current pulse there is a significant population of Xe III. The overionized plasma results in an increased electron density and, therefore, a larger phase mismatch between the driving laser pulse and the generated harmonics.

Spectroscopic measurements and model calculations with a hydrodynamic/atomic physics code developed in house [22], show that the discharge plasma is completely ionized in the center region of the capillary at a time delay of 2 us, corresponding to the observed maximum of the harmonic signal. The model is consistent with the relative $\mathrm{XeI}, \mathrm{Xe}$ II, and Xe III line intensity measurements of Fig. 4(b). In contrast to the spectroscopy of the pulsed discharge, the spectrum of the plasma created by the small simmer current shows very strong Xe I lines, with weaker $\mathrm{Xe}$ II and Xe III lines. Finally, calculation of wellestablished Ammosov-Delone-Krainov strong-field ionization rates show that all harmonics from xenon gas at energies $>75 \mathrm{eV}$, are produced under conditions where the neutral xenon has already been completely ( $>99 \%)$ depleted [23]. These results show that the highest energy harmonics observed were generated exclusively from ions.

In conclusion, we have demonstrated that harmonic generation from xenon ions in a capillary discharge plasma can significantly extend the highest observable harmonic to shorter wavelengths by a factor of 2 compared with previous measurements. We have observed an extension of the highest observable harmonic by $\sim 75 \mathrm{eV}$ over previous experiments, as well as an enhancement of the flux of the harmonics in the $90 \mathrm{eV}$ region by nearly 2 orders of magnitude. The presence of the discharge reduces phase modulation of the driving laser and increases the laser intensity available for harmonic generation. In the future, the combination of a multiply ionized discharge plasma waveguide [17] with HHG quasi-phase-matching techniques [24] has the potential to enhance efficient harmonic generation at much shorter wavelengths.

The authors would like to acknowledge support from the U.S. Department of Energy, Chemical Sciences, Geosciences, Biosciences Division of the Office of Basic Energy Science, and by the Engineering Research Centers Program of the National Science Foundation under NSF Contract No. EEC-0310717. B. C. W. acknowledges support from JILA.

[1] M. Bauer, J. Phys. D 38, R253 (2005).

[2] R. A. Bartels et al., Science 297, 376 (2002).

[3] R. Velotta et al., Phys. Rev. Lett. 87, 183901 (2001).

[4] J. Itatani et al., Nature (London) 432, 867 (2004).

[5] A. McPherson et al., J. Opt. Soc. Am. B 4, 595 (1987).

[6] M. Ferray et al., J. Phys. B 21, L31 (1988).

[7] K. C. Kulander, K. J. Schafer, and J. L. Krause, in SuperIntense Laser-Atom Physics, edited by B. Piraux, A. L'Huillier, and K. Rzazewski (Plenum, New York, 1993), Vol. 316, p. 95.

[8] M. Lewenstein et al., Phys. Rev. A 49, 2117 (1994).

[9] A. L'Huillier et al., Phys. Rev. A 46, 2778 (1992).

[10] S. C. Rae, Opt. Commun. 97, 25 (1993).

[11] E. A. Gibson et al., Phys. Rev. Lett. 92, 033001 (2004).

[12] J. Seres et al., Nature (London) 433, 596 (2005).

[13] C. G. Wahlström et al., Phys. Rev. A 48, 4709 (1993).

[14] H. M. Milchberg, C. G. Durfee, III, and T. J. McIlrath, Phys. Rev. Lett. 75, 2494 (1995).

[15] A. Butler, D. J. Spence, and S. M. Hooker, Phys. Rev. Lett. 89, 185003 (2002).

[16] Y. Wang et al., Phys. Rev. E 72, 026413 (2005).

[17] B. M. Luther et al., Phys. Rev. Lett. 92, 235002 (2004).

[18] A. B. Fedotov et al., J. Opt. Soc. Am. B 8, 363 (1991).

[19] Y. Akiyama et al., Phys. Rev. Lett. 69, 2176 (1992).

[20] C. G. Wahlström et al., Phys. Rev. A 51, 585 (1995).

[21] H. Wang et al., J. Opt. Soc. Am. B 16, 1790 (1999).

[22] M. Berrill and J. J. Rocca (unpublished).

[23] M. V. Ammosov, N. B. Delone, and V. P. Kraĭnov, Sov. Phys. JETP 64, 1191 (1986).

[24] E. A. Gibson et al., Science 302, 95 (2003). 